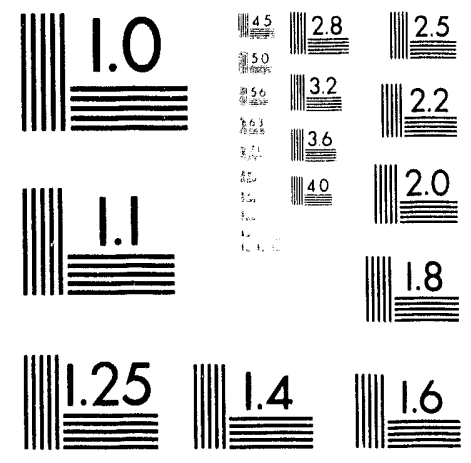



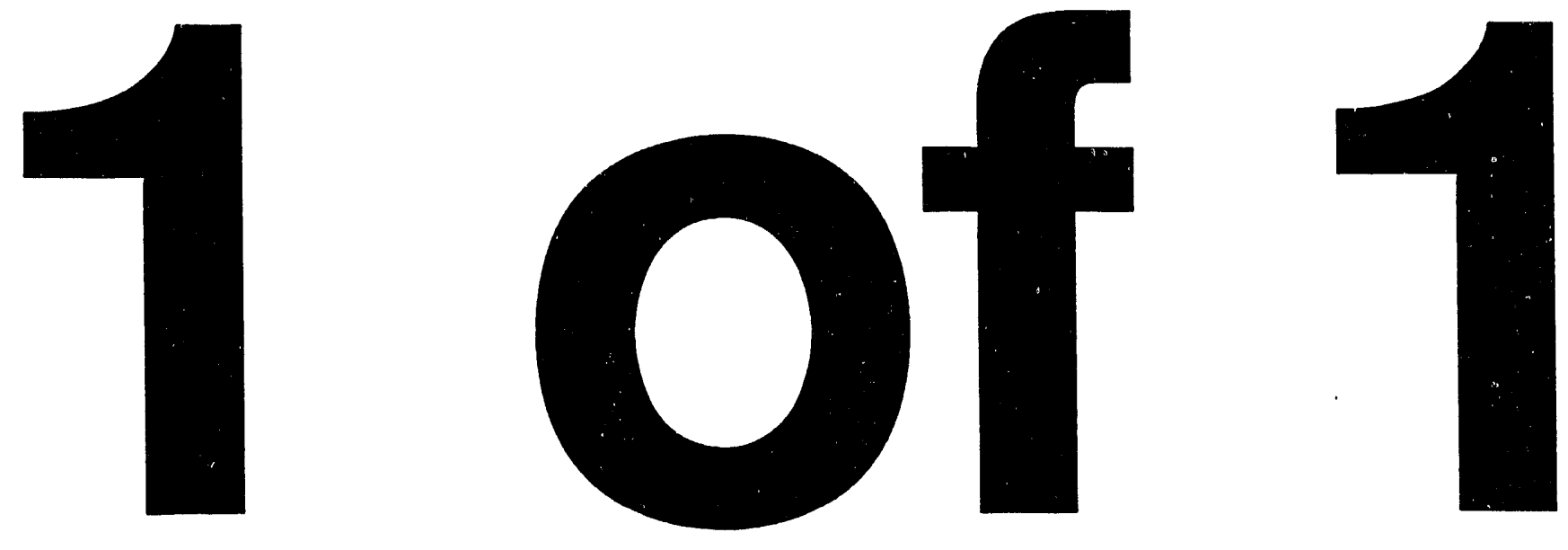
LBL-34168

UC-412

\title{
Cratering Rates from Lunar Xenospherules
}

\author{
Richard A. Muller \\ Lawrence Berkeley Laboratory and Department of Physics \\ University of California \\ Berkeley, California 94720
}

May 1993

This work was supported by the Director, Office of Energy Research, Office of High Energy and Nuclear Physics, High Energy Physics Division, of the U.S. Department of Energy under Contract No. DE-AC03$76 \mathrm{SF} 00098$.
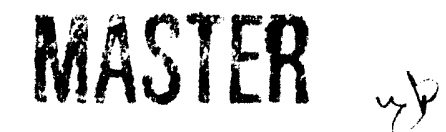
Richard A. Muller

25 May 1993

\section{Introduction}

The goal of this project is to obtain the time history of impacts on the moon, with emphasis on recent impacts. In particular, the project could prove (or disprove) the existence of comet storms and provide the dates when they occurred.

\section{Background:}

In 1980, Luis and Walter Alvarez, Frank Asaro and Helen Michel published the hypothesis that the impact of a comet or asteroid on the Earth was the cause of the Cietaceous catastrophe, the mass extinction that killed over $50 \%$ of the Eath's life, including the dinosaurs. The 'smoking gun' for this event has now been found, with the identification of the Chicxulub crater in the Yucatan as the site of the impact ${ }^{1}$, and its dating to precisely the time of the extinctions, to within $0.1 \mathrm{Myr}$. The dating was done at the local Geochronology Center operated by the Institute for Human Origins. It used a newly developed technique of ${ }^{39} \mathrm{Ar} / 40 \mathrm{Ar}$ dating (a refined method of $\mathrm{K} / \mathrm{Ar}$ dating) that uses a laser to step heat a sample of glass. The samples used were 'spherules,' glassy splash from the impact, with diameters of about 500 microns, and weighing only 0.2 to $0.3 \mathrm{mg}$

Shortly after the Nemesis hypothesis was proposed, we realized that the effect of a comet storm might be to give an illusion of an extended extinction period. ${ }^{2}$ A comet storm occurs when the passage of a nearby star (or of a companion star, such as Nemesis) passes close to the Oort comet cloud, and perturbs the orbits to fill the normally empty 'eye' of the comet storm, the inner solar system, with approximately $10^{9}$ comets. A series of impacts taking place in a 1-million year period would give a series of extinctions, "stepwise", according to a later terminology, that, if not recognized, would look like a gradual extinction over the same period. Soon afterwards several extinctions were analyzed according to this model, and the stepwise extinction pattern was seen as reasonable. ${ }^{3}$

${ }^{1}$ C. Swisher et al., Science vol 257, 954, [14 Aug 1992].

2R. A. Muller, "Evidence for a Solar Companion Star," Proceedings of the International Astronomical Union Symposium 112, "The Search for Extraterrestrial Life, Recent Developments," June 18-21, 1984 (D. Reideel Pub. Co, Dordrect Holland); also published as Lawrence Berkeley Laboratory preprint LBL-18271 (1984).

${ }^{3}$ Hut et al., Nature vol 329, Sept 1987, pg 118-126. 
The present estimate for cratering rates, based on analysis of lunar craters, is shown in the following figure based on a paper by Hartmann (1972; see Glass p. 226). This curve is based on cratering densities seen in objects of known ages (e.g. Mare Tranquillitatis has an age of $3.6-3.7 \times 10^{9} \mathrm{yr}$; Copernicus of $0.9 \times 10^{9} \mathrm{yr}$, and Tycho of $0.27 \times 10^{9} \mathrm{yr}$.).

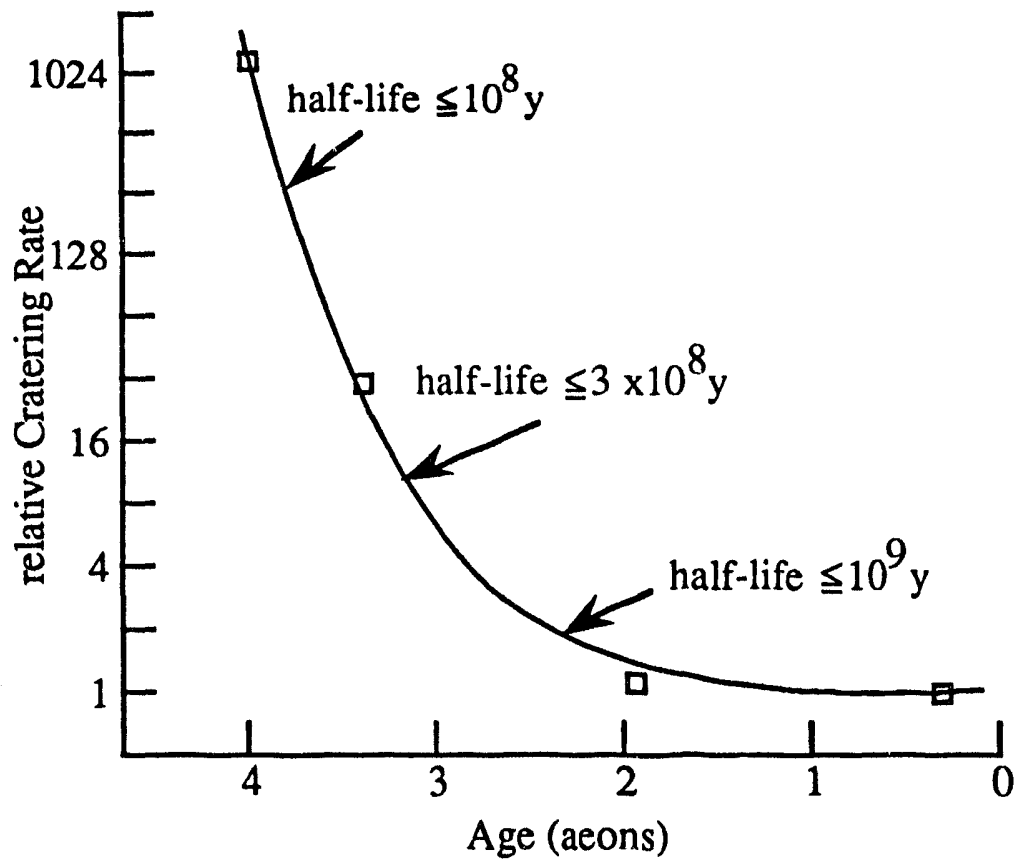

Estimated Cratering history of the Moon

Our first goal is simply to improve on this plot. Our second goal is to see structure, such as peaks that would be due to comet storms.

Evidence for comet storms has been found in the nature of the extinctions themselves 4 in the periodicity of cratering on the earth ${ }^{5}$ and elsewhere ${ }^{6}$ but this evidence is circumstantial at best, and has been disputed. ${ }^{7}$ The comet storm hypothesis has recently received some support from the discovery that two of the largest craters on the earth, the Chicxulub crater in Mexico and the Manson crater in Iowa, occurred within 200,000 years of each other.

Comet storms on the Earth will also hit the moon; although the number of impacts will be smaller (the moon has only $7.4 \%$ of the Earth's surface area) the record is much better kept, since except for impacts the surface of the Moon has been virtually undisturbed for over four billion years. If the comet storms are accompanied by large numbers of small objects (in addition to the $1 \mathrm{~km}$ comets) then a record of them could have been left on the moon.

${ }^{4}$ Hut et al., Nature vol 329, Sept 1987, pg 118-126.

${ }^{5}$ W. Alvarez and R. Muller, Nature 308, $718-720$ (1984)

${ }^{6}$ S. Perlmutter and R. Muller, Icarus.74, 369-373 (1988).

${ }^{7}$ The periodicity of the cratering has been disputed, for example, by Shoemaker and Wolf. 
In this paper we outline a method that could allow the dating of hundreds to thousands of lunar craters without the need for further lunar expeditions, using samples that have already been collected in the Apollo missions. In addition to finding evidence for individual comet storms, the experiment should show the long-term trends in cometary impacts. I believe that it will not determine absolute average cratering rates with higher precision than is already known.

One of the most interesting result that we can anticipate would be the discovery of clusters of impacts, perhaps one centered about the time 65 Myr ago, or 250 Myr ago at the time of the Permian-Triassic extinctions. Perhaps we could eventually see a series of such events, separated in time by the 26 Myr that paleonotologists Raup and Sepkoski have found in the mass extinctions on the Earth. ${ }^{8}$ However we do not know whether the senstivity necessary to resolve such events can be achieved from single spherules.

\section{Proposed experiment:}

Virtually every sample of lunar soil contains glass spherules that come from impacts on the lunar surface. These are droplets of suface material that melted in the impact, were thrown from one to hundreds of kilometers or more, and solidified (by radiative cooling) before they landed. According to B. Glass", "It is generally possible to recover several hundred glass spherules $>100$ micron diameter from a 0.5 to 1.0 gram size soil sample."

It is known (in particular, from the rays of craters such as Tycho and Copernicus) that some crater debris is thrown very far. This is reasonable, since typical impact velocities are about $45 \mathrm{~km} / \mathrm{sec}$, much greater than the lunar escape velocity of $2.38 \mathrm{~km} / \mathrm{sec}$.

We hypothesize that some of these spherules come from distant impacts, and therefore that a single soil sample contains a record of many lunar events. This hypothesis will be tested in the proposed pilot experiment.

A relatively recent improvement in radioisotope dating allows the time of the impacts to be measured with remarkable precision. For terrestrial samples younger than 0.1 aeon ${ }^{10}$, accuracies better than better than \pm 0.2 million years have been obtained from single spherules. For the success of this project it is not necessary to duplicate such precision; indeed, accuracies of several million years will give important information on cratering rates. However one of the goals of this pilot project is to see what precision can be obtained with present equipment.

The equipment necessary to do such dating is available at the Geochronology Center of the Institute for Human Origins in Berkeley. I spoke to Garniss Curtis about collaborating

\footnotetext{
${ }^{8}$ Raup, D. M. and Sepkoski, J.J. , Proc. Nat. Acad. Sci. USA v 81, $801-805$ (1984).

${ }^{9}$ B. P. Glass, Introduction to Planetary Geology, 1982.

${ }^{10}$ For the physicist reader, one aeon $=10^{9}$ year $=1 \mathrm{Gyr}$.
} 
on this project, and he was enthusiastic. He enlisted the help of the director of the laboratory, Paul Renne, and scientific staff member Carl Swisher.

Our immediate interest will be in recent spherules (i.e. younger than 3 aeons) from distant craters. There are several morphological features that will help us select younger spherules, including absence of micro-impact pits on their surfaces, no cavities, no splashed silicate glass. 6 We may wish to pick sites in the lunar maria, with low numbers of local craters. An electron microprobe is available for our use that can be used to chose spherules which are different from each other, and different from the main mass of spherules (which probably are local in origin). We like to refer to the odd spherules, the ones that appear different from the local material, as xenospherules, from the root xeno meaning "strange."

We have requested from NASA one to two grams of lunar soil, to be chosen with their advice; from this material, up to 100 spherules will be extracted and examined, by microscopy, SEM, and electron microprobe. Up to twenty five of these will chosen, based on their dissimilarity from each other, for ${ }^{39} \mathrm{Ar} / 0 \mathrm{Ar}$ and ${ }^{37} \mathrm{Ar} /{ }^{38} \mathrm{Ar}$ cosmic ray exposure age dating. We would like to find 25 spherules from 25 separate origins. Our goal is to determine whether the rate of impacts on the moon has been constant over the last 3 aeons. We will not obtain the absolute rate; rather we will measure the relative rate over this period.

From physical appearance and microprobe analysis we hope to select spherules that are relatively young, different from each other, and originate from impacts rather than volcanic events. Thus we hope to obtain the ages of 25 distinct (although unidentified) lunar craters. There may be some value in picking spherules that are rich in silica, since (according to Glass' text, ${ }^{1}$ p. 217), they are "similar to interstitial glasses in mare basalts." If we choose spherules that originated in the mare basalts, then we are deemphasizing the many early craters that represent the heavy bombardment that took place prior to the flooding of the lunar plains. Other chemical and geophysical methods for distinguishing recent spherules will be developed.

In addition we plan to use procedures in spherule selection that have been developed to increase the accuracy of age determination. These have been summarized by Spangler et al. ${ }^{11}$, and include: clarity, lack of solar wind pitting and etching, lack of devitrification. Naturally all such procedures can introduce biases that could simulate slow drifts in cratering rates; however it is less likely that they would simulate a short period of high intensity impacts, as in a comet shower.

This project is seen as a pilot study to demonstrate the value of the measurement of much larger numbers of spherules. In the future we envision similar measurements of thousands of spherules.

\footnotetext{
${ }^{11}$ Proc. 14 Lunar \& Planetary Sci Cof, part 2, JGR vol 89, p. B487-97, 1984
} 


\section{Lateral transport:}

One of the key questions is how many of the spherules we find come from distant regions, and whether we can separate them. H. J. Melosh, in his book "Impact Cratering," 1989, says "lateral transport by meteoritic impact is not very efficient." p 197. However "large, rare impact events [could] disperse small amounts of material." A small amount of what is believed to be Tycho ejecta was found in Apollo 17 samples; a fragment of Copernicus at Apollo 15. $(201,202)$. From chart on page 202 of Melosh's text, we can estimate that $10^{-3}$ of regolith accumulation comes from ranges greater than $100 \mathrm{~km}$, in a model that neglects secondary ejecta and rays of large distant impacts. Our hope is that a larger fraction of the spherules (which by their nature are the small droplets that carry far) are from distant craters. Since we are only looking for roughly 25 different craters, we only need to sample spherules from craters that are within $10-50 \mathrm{~km}$ of the site. Of course the nearby craters are likely to be more chemically homogeneous than if they come from a variety of distant locations.

\section{Sample selection:}

We solicit the advice of NASA on the selection of soil samples for the successful completion of the pilot project. We expect that the following criteria will be useful:

(1) We do not necessarily want a sample that contains large numbers of spherules, for that may indicate the presence of a nearby event, particularly if the dominant spherules are known to be chemically or physically similar. We want to date roughly 25 spherules from 25 different events, and the spherules do not have to be abundant.

(2) It may be better to chose a sample from the lunar maria rather than from the highlands, since in the maria most spherules from old impacts will have been buried; thus it will be easier to choose spherules from recent impacts. We are not particularly interested in rediscovering the late heavy bombardment; our interest is in recent ( $<3 \mathrm{ae}$ ) impacts. (Only in astrophysics could we call 3 ae recent!)

(3) We probably want relatively shallow samples, from the upper few centimeters of the soil, again to emphasize recent craters.

Based on the above criterion, soil samples from Apollo 11 will be considered. We understand that there were many such samples taken with minimum documentation from this landing, and that the landing site was chosen to be relatively crater-free. Moreover, these samples are not currently under heavy demand because of the relative lack of documentaion. 
(We were told that the astronauts stuffed extra soil in the spaces of between the collected moon rocks.)

There may be a better choice of regolith available; for example, soil collected at a site that is relatively free of very close craters, but has a large number of young craters within 10 to $50 \mathrm{~km}$. As we mentioned earlier, we solicit advice of the soil selection.

\section{Instrumentation}

For physical and chemical analysis we will make use of the Electron Microprobe Analysis Laboratory in the Department of Geology and Geophysics at the University of California at Berkeley. Collaborating on this experiment is Walter Alvarez from that department, and graduate student Timothy Culler.

The electron microprobe uses 5-30 keV electrons to induce emission of xrays in the range 0.1 to $15 \mathrm{keV}$. Its specifications are as follows:

- ARL SEMQ electron microprobe with 8 wavelength spectrometers

- Analytical range from Be to $\mathrm{U}$; typical volume 1 - 10 microns

- Analytical sensitivity from $100 \%$ to $50 \mathrm{ppm} \pm 2 \%$

- analysis time $10 \mathrm{sec}$ (major elements) - $300 \mathrm{sec}$ (traces)

Measurements of the ${ }^{39} \mathrm{Ar} /{ }^{40} \mathrm{Ar}$ solidification age and ${ }^{38} \mathrm{Ar} /{ }^{37} \mathrm{Ar}$ cosmic ray ages will be made at the Geochronology Center of the Institute for Human Origins at Berkeley. Numerous Ar/Ar dates have been obtained at this center, including the recent measurment of the age of the Chixulub crater (in which an accuracy of \pm 0.1 Myr was obtained.)

The Geochronology Center has two fully automated extraction line/mass spectrometer systems use for high-precision ${ }^{40} \mathrm{Ar} /{ }^{39} \mathrm{Ar}$ dating. The first uses a Macintosh II computer to control a rampable $6 \mathrm{~W}$ continuous Ar-ion laser, actuator-driven $x-y$ stage and sample chamber; Ar purification system; and a low-blank Mass Analyzer Products 215 nobel-gas mass spectrometer. Typical sample quantities are ten to a hundred micrograms. The second system employs a Nd-YAG laser and resistance furnace options for Ar extraction, and a Mass Analyzer Products 215-50 mass spectrometer. The systems are currently producing between 100 and 200 dates per year. High precision results with standard errors of the mean are typically as low as $0.4 \%$ for 0.2 Ma sanidines, $<0.2 \%$ for samples of Pliocene age, and as low as $0.05 \%$ for $20 \mathrm{Ma}$ or older $\mathrm{K}$-fledspars. The high precision is obtained because of many factors, including the high sensitivity of the MAP 215 mass spectrometer; computercontrolled, highly reproducible analytical condition, low blank correction from extraction line and mass spectrometer (M/e $36<1 \times 10^{-18}$ moles, M/e $<1 \times 10^{-16}$ moles; techniques to filter electronic noise, analyze long-term blank and baseline trends, and automated curve-fitting procedures. 
Lunar samples, of course, require corrections for spallation and the possible implantation of argon from solar wind. We will measure all isotope masses from mass 36 through 40 and plan to use isotope correlation diagrams to help analyze solar wind, spallation, and other trapped components.

\section{Prior work:}

The ability to date individual spherules by ${ }^{39} \mathrm{Ar} /{ }^{40} \mathrm{Ar}$ dating became available for the first time in the late 1970s, when Huneke at Cal Tach measured five glass spherules. ${ }^{12}$ His interest was in finding spherules from a local impact, and they were chosen from the lunar soil because they matched other material known from the crater of interest. Several other papers in which spherules were measured ${ }^{13}$ followed this same pattern; apparently because of the difficulty of measuring many samples, very special spherules were always chosen. In the late 1980s and early 1990s interest in measuring spherules waned, just as the ability to measure large numbers of individual spherules became practical. We have found no example in the literature of ${ }^{39} \mathrm{Ar} / 40 \mathrm{Ar}$ dating of "xenospherules."

Scientific team: The following scientists have agreed to collaborate on this project:

Garniss Curtis, professor of Geology and Geophysics at U.C. Berkeley.

Kunihiko Nishiizumi, Space Sciences Laboratory, University of California at Berkeley

Paul Renne, Geochronology Center, Berkeley

Carl Swisher, Geochronology Center, Berkeley

Walter Alvarez, Professor of Geology and Geophysics, Univ. Calif. Berkeley

Tim Culler, Graduate Student in Geology and Geophysics, Univ. Calif. Berkeley

\section{Conclusions}

By the end of this year the prospects are good that we can obtain the ages of 25 lunar craters by examination of their ejected microspherules. From this we can get a crude estimate of relative crating rates, and possibly see signs of comet storms in enhanced cratering activity. We will also determine whether we should proceed to a larger study, perhaps studying severa hundred to several thousand spherules.

\footnotetext{
12Proceedings of the 9th Lunar and Planetary Science Conference; publ. 1979

13 c.g. E. C. Alexander et al., 11 th Lunar and Planctary Science Conference, 1980; also Ar-39 Ar-40 ages for the Apollo 15 Green and Yellow Volcanic Glasses, by R. R. Spangler, R. Warasila, J. W. Delano, in Proc. of 14th Lunar \& Planetary Sci Conf, part 2, JGR vol 89 Suppl, p B487-B497, Feb 15, 1984. [Used laser microprobe on glassy samples $100-750$ micron diameter. Ages obtained were 0.275 to 3.62 acons. Spangler is at Stony Brook.]
} 

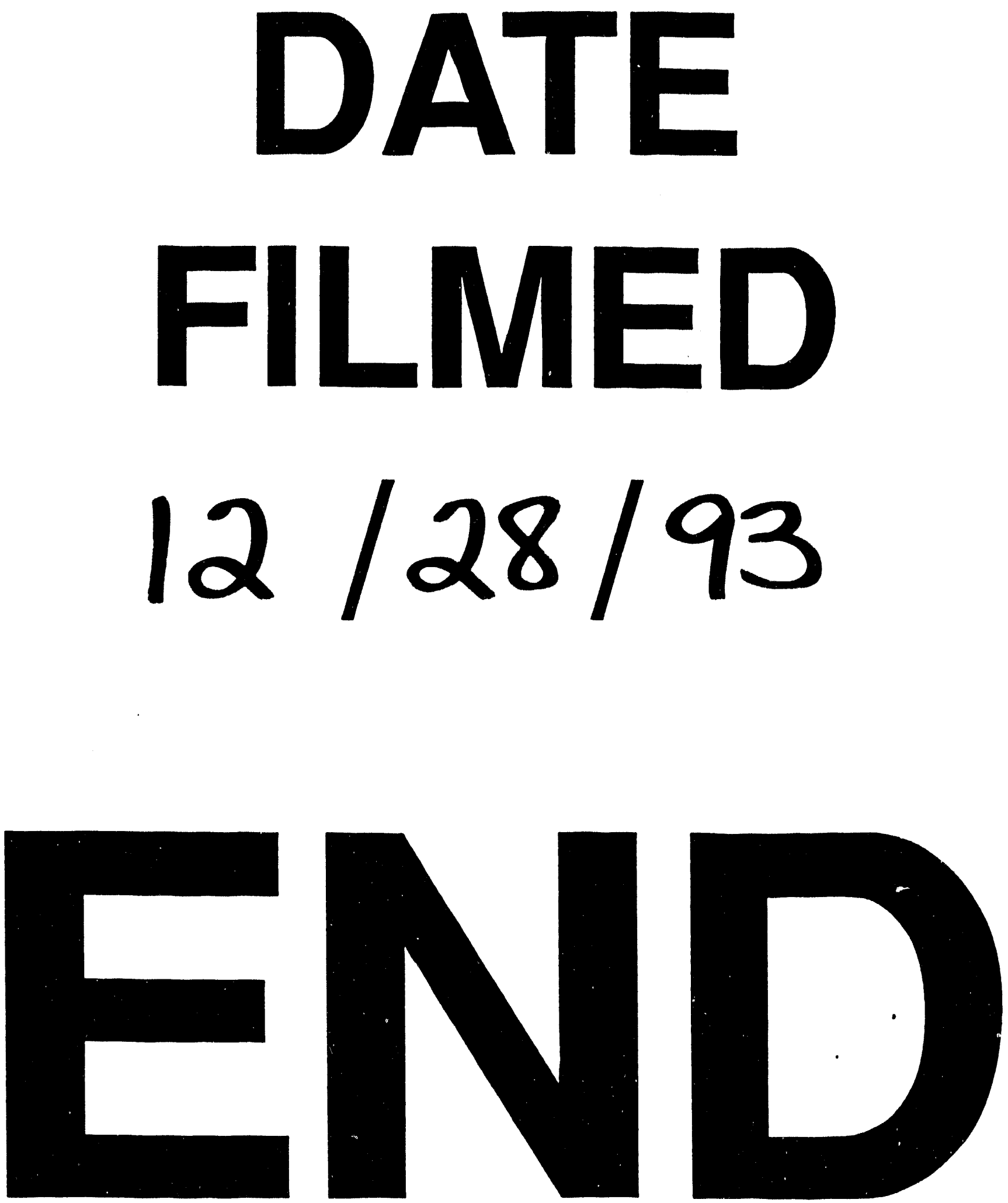
